\title{
Polarized superconductors in nanostructures
}

Gerd Bergmann, Jia G. Lu, and Robert Mueller

Citation: Journal of Applied Physics 99, 054314 (2006);

View online: https://doi.org/10.1063/1.2177930

View Table of Contents: http://aip.scitation.org/toc/jap/99/5

Published by the American Institute of Physics

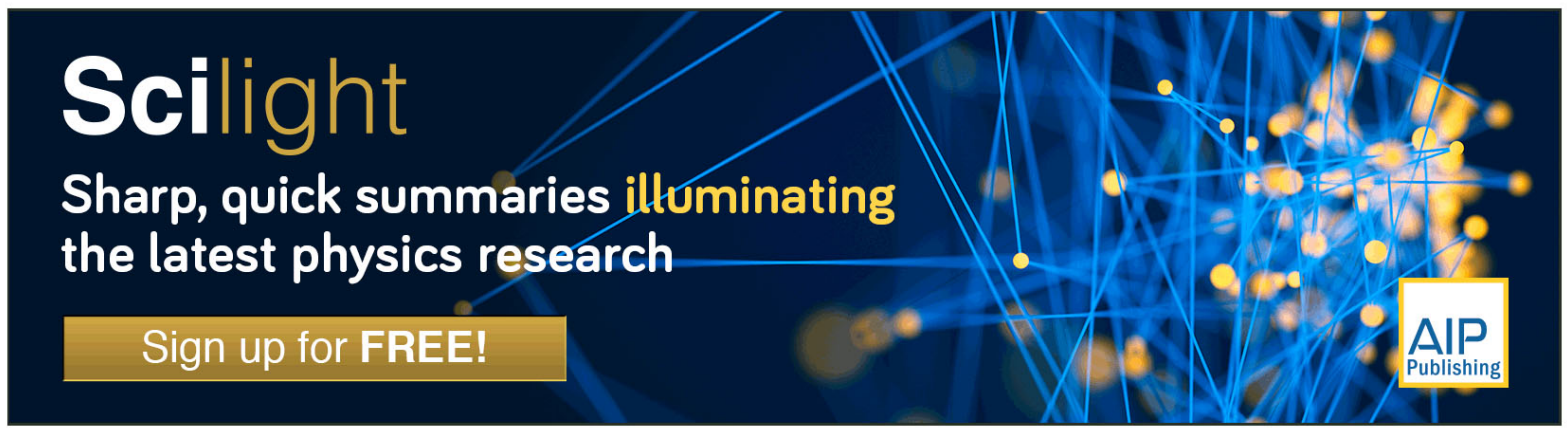




\title{
Polarized superconductors in nanostructures
}

\author{
Gerd Bergmann ${ }^{\text {a) }}$ \\ Department of Physics, University South California, Los Angeles, California 90089-0484 \\ Jia G. Lu ${ }^{\text {b) }}$ \\ Departments of Materials Science and Electrical Engineering, University of California, \\ Irvine, California 92697 \\ Robert Mueller \\ IFF der FZ-Jülich, Leo Brandt Strasse, D-52425 Jülich, Germany
}

(Received 11 July 2005; accepted 26 January 2006; published online 14 March 2006)

\begin{abstract}
Single electron transistors with two ferromagnetic electrodes in the antiferromagnetic alignment and a superconducting island can induce spin polarization in the superconductor. The effect of spin polarization on the superconducting properties is studied theoretically. Spin injection is an interesting alternative to high magnetic fields to induce large spin polarization in a superconductor. Since this method is not restricted to extremely thin superconductors, it provides a mean to verify exotic states in $s$ superconductors, such as the Fulde-Ferrel-Larkin-Ovchinnikov state. The combination of spin injection and nanosized islands promises an approach to investigate superconductors with high spin polarization. (C) 2006 American Institute of Physics.
\end{abstract}

[DOI: $10.1063 / 1.2177930]$

In recent years the technique of single electron transistors with ferromagnetic electrodes and a superconducting island has made it possible to inject one spin orientation into the superconducting island and extract the other spin orientation. ${ }^{1-6}$ The tunneling properties of such a polarized superconductor have been investigated theoretically. ${ }^{7,8}$ In this paper, we want to demonstrate that a superconductor with an imposed finite spin polarization can have rather interesting properties.

Superconductors with spin polarization have been intensively investigated in the past. ${ }^{9-13}$ In these experimental and theoretical investigations the polarization was imposed by an external magnetic field and the superconductor was in thermal equilibrium. In contrast, in single electron transistors with injected spin polarization the spin up and down electrons are not in equilibrium with each other.

A typical single-electron transistor (SET) in these experiments consists of a superconducting $\mathrm{Al}$ film with dimensions of the order of 1-2 $\mu \mathrm{m}$ length, 50-100 nm width, and $20-40 \mathrm{~nm}$ thickness. The $\mathrm{Al}$ is oxidized and crossed by two narrow ferromagnetic Co strips. These form two tunneling junctions with the $\mathrm{Al}$ which are separated by $0.5 \mu \mathrm{m}$ or less. The magnetization of the two Co films are aligned antiparallel. When a current is passed through the two junctions and the Al film, the polarization of the injected and the extracted current is opposite and electrons of one spin orientation are accumulated in the $\mathrm{Al}$ film. This spin accumulation can be achieved in zero magnetic field. Although the $\mathrm{Al}$ island is small its superconductivity can still be described by the Bardeen-Cooper-Schrieffer (BCS) theory, confinement effects are not yet important. This is demonstrated by the dependence of the transition temperature on a parallel magnetic

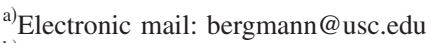

${ }^{b)}$ Electronic mail: jglu@uci.edu
}

field, the tunneling $I-V$ curve in SETs consisting of an Al island and two $\mathrm{Al}$ electrodes, the observation of the Coulomb blockade with charge units of two electrons, etc. ${ }^{14-16}$ Strong one-dimensional deviations from BCS theory occur at much narrower film strips, such as carbon nanotubes covered with thin films of superconductors. ${ }^{17}$

In this paper, we consider the effect of spin injection on the properties of the superconducting island. It is assumed that the spin-orbit interaction is small and therefore the lifetime of the spin polarization is long and the spatial distribution of the polarization is homogeneous. This condition appears to be fulfilled in these small $\mathrm{Al}$ islands as shown by Jedema et $_{\mathrm{al}}{ }^{3}$ who observed precession of the injected spins in the $\mathrm{Al}$ island.

We describe the polarization $m$ by the dimensionless parameter $\beta$ so that $m=\left(n_{\uparrow}-n_{\downarrow}\right)=\beta N k_{B} T_{s}$ (where $n_{\uparrow}, n_{\downarrow}$ are the electron densities of spin up and down electrons, $T_{s}$ is the transition temperature of the superconducting island, and $N$ is the BCS density of states of the island in the normal conducting state).

In Fig. 1 the superconducting and the normal ground state (at $T=0)$ are sketched for a finite spin polarization. The abscissa gives the occupation number for spin up and down while the ordinate gives the (free electron) band energy $\varepsilon_{\mathbf{k}}$ measured from the Fermi energy (not the quasiparticle energy $E_{\mathbf{k}}$ ). In the normal state the Fermi energy has different values for the spin up and down electrons. For a given value of $\beta$ this shift is $\mu_{\uparrow}-\mu_{\downarrow}=\beta k_{B} T_{s}$. For the superconducting state the pair states within $\pm \beta k_{B} T_{s} / 2$ of the Fermi energy $\varepsilon_{\mathrm{F}}$ are blocked by the spin up electrons and only the remaining states for $\left|\varepsilon_{\mathbf{k}}\right|>\beta k_{B} T_{s} / 2$ are occupied with the pair probability $h_{\mathbf{k}}$ (using the original BCS nomenclature).

The BCS theory (Ref. 18) expressed the superconducting state in terms of the pair-state occupation $h_{\mathbf{k}}$ and the quasiparticle occupation $f_{\mathbf{k}}$. If one adds to their free energy the 


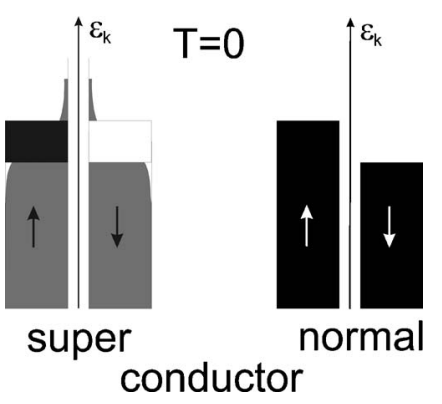

FIG. 1. Electron distribution for a metal with finite electron polarization in the superconducting and normal states. The light shaded region in the superconductor represents occupation with $\left(\mathbf{k}_{\uparrow},-\mathbf{k}_{\downarrow}\right)$-electron pairs, the dark spin up region is occupied with single electrons, having the $\mathbf{k}_{\uparrow}$ occupied, and the white region $-\mathbf{k}_{\downarrow}$ is empty.

term $\lambda\left[\Sigma_{\mathbf{k}}\left(f_{\mathbf{k} \uparrow}-f_{\mathbf{k} \downarrow}\right)-m\right]$ as a condition of constant electron polarization ( $\lambda$ is the LaGrange parameter) then one obtains in complete analogy to BCS's minimization of the free energy the results

$$
\begin{aligned}
& f_{\mathbf{k} \uparrow, \downarrow}=\left[\exp \left(\frac{E_{\mathbf{k}} \mp \lambda}{k_{B} T}\right)+1\right]^{-1}, \\
& h_{\mathbf{k}}=\frac{1}{2}\left(1 \mp \frac{\varepsilon_{\mathbf{k}}}{E_{\mathbf{k}}}\right),
\end{aligned}
$$

and the self-consistent equations for the gap $\Delta(T)$ and the LaGrange parameter $\lambda$ (see also Ref. 7)

$$
\begin{aligned}
& 1=V N \int_{0}^{k_{B} \Theta_{D}} \frac{1}{E_{\mathbf{k}}}\left[1-\left(f_{\mathbf{k} \uparrow}+f_{\mathbf{k} \downarrow}\right)\right] d \varepsilon_{\mathbf{k}}, \\
& 2 N \int_{0}^{k_{B} \Theta_{D}}\left(f_{\mathbf{k} \uparrow}-f_{\mathbf{k} \downarrow}\right)=m=\beta N k_{B} T_{s},
\end{aligned}
$$

where $E_{\mathbf{k}}=\sqrt{\Delta^{2}(T)+\varepsilon_{\mathbf{k}}^{2}}$ is the quasiparticle energy, $N$ is the BCS density of states, $V$ represents the BCS interaction, and $\Theta_{D}$ the Debye temperature.

1)

At zero temperature the gap equation becomes (see Fig.

$$
1=V N \int_{\beta k_{B} T_{s} / 2}^{k_{B} \Theta_{D}} \frac{1}{\sqrt{\Delta_{0}^{2}+\varepsilon^{2}}} d \varepsilon,
$$

yielding the solution for the gap $\Delta_{0}$ at zero temperature and finite polarization $\beta$

$$
\Delta_{0}=\sqrt{\Delta_{00}\left(\Delta_{00}-\beta k_{B} T_{s}\right)},
$$

where $\Delta_{00}$ is the gap at zero temperature and zero polarization. The superconductivity disappears at zero temperature when $\beta$ reaches the famous gap ratio.

$$
\beta=\frac{\Delta_{00}}{k_{B} T_{s}}=1.75 .
$$

At finite temperature one has to iterate the self-consistent equations (3) and (4). In Fig. 2 the temperature and polarization-dependent energy gap $\Delta(T)$ is shown as a function of temperature for different polarization parameters $\beta$. Only the gap curve for $\beta=0$ has its maximum at zero temperature. For finite polarization parameter $\beta$ the maximum of

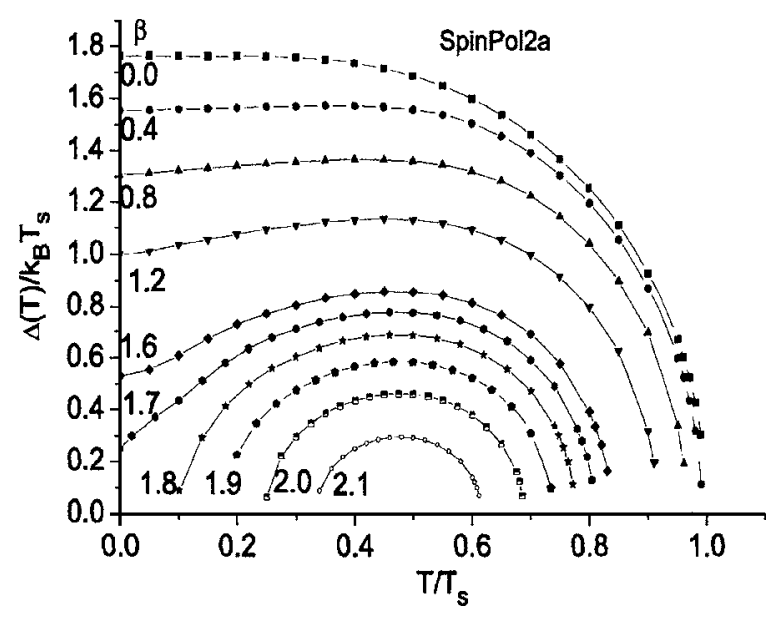

FIG. 2. The energy gap $\Delta(T) / k_{B} T_{s}$ as a function of temperature for different imposed spin polarizations $\beta$. For a finite range of $\beta>\Delta_{00} / k_{B} T_{s}$ superconductivity is destroyed at zero temperature but exists at finite temperature.

the gap moves towards half the transition temperature. For $\beta>\Delta_{00} / k_{B} T_{s}$ one has an island of superconductivity. As a function of temperature one has two transition points and at sufficiently low temperature the normal state is stable.

The dependence of $\Delta(T, \beta)$ on the polarization parameter $\beta$ corresponds to an extremum in the free energy of the superconducting state. To ensure that its free energy lies below the free energy of the corresponding normal state with the same polarization we calculate the difference of the two free energies $F_{s}-F_{n}$.

The free energy of the superconducting state is given in terms of $\Delta, h_{\mathbf{k}}$, and $f_{\mathbf{k} \sigma}(\sigma=\uparrow$ or $\downarrow)$ by

$$
\begin{aligned}
F_{s}= & 2 N \int_{0}^{k_{B} \Theta_{D}} d \varepsilon_{k} \varepsilon_{k}\left[2 h_{k}\left(1-f_{\mathbf{k} \uparrow}-f_{\mathbf{k} \downarrow}\right)+f_{\mathbf{k} \uparrow}+f_{\mathbf{k} \downarrow}\right] \\
& -N \frac{[\Delta(T)]^{2}}{N V}+2 k_{B} T N \\
& \times\left[\sum_{\sigma} \int_{0}^{k_{B} \Theta_{D}} f_{\mathbf{k} \sigma} \ln \left(f_{\mathbf{k} \sigma}\right)+\left(1-f_{\mathbf{k} \sigma}\right) \ln \left(1-f_{\mathbf{k} \sigma}\right) d \varepsilon_{k}\right] .
\end{aligned}
$$

The free energy of the normal conductor with finite polarization $\beta k_{B} T_{s}$ is

$$
F_{n}=N\left[-\frac{\pi^{2}}{3}\left(k_{B} T\right)^{2}+\frac{1}{4}\left(\beta k_{B} T_{s}\right)^{2}\right] .
$$

In Figs. 3(a) and 3(b) the difference between the superconducting and the normal free energies $F_{s}-F_{n}$ in the units of $N\left(k_{B} T_{s}\right)^{2}$ is plotted as a function of temperature for different polarization parameters $\beta$. Only for $\beta=0$ does the minimum of $F_{s}-F_{n}$ lie at zero temperature. For finite $\beta$ the minimum moves towards a finite temperature. The superconducting state is stable for the whole range of $(T, \beta)$ in which we obtained a finite energy gap.

We can construct a phase diagram in the $t-\beta$ plane where $t=T / T_{s}$. The boundary between the superconducting and the normal state is given by the condition that $\Delta=0$. Then the Fermi distribution in Eq. (1) becomes also the Fermi distribution for the normal conducting phase. As a consequence the chemical potentials of spin up and down 

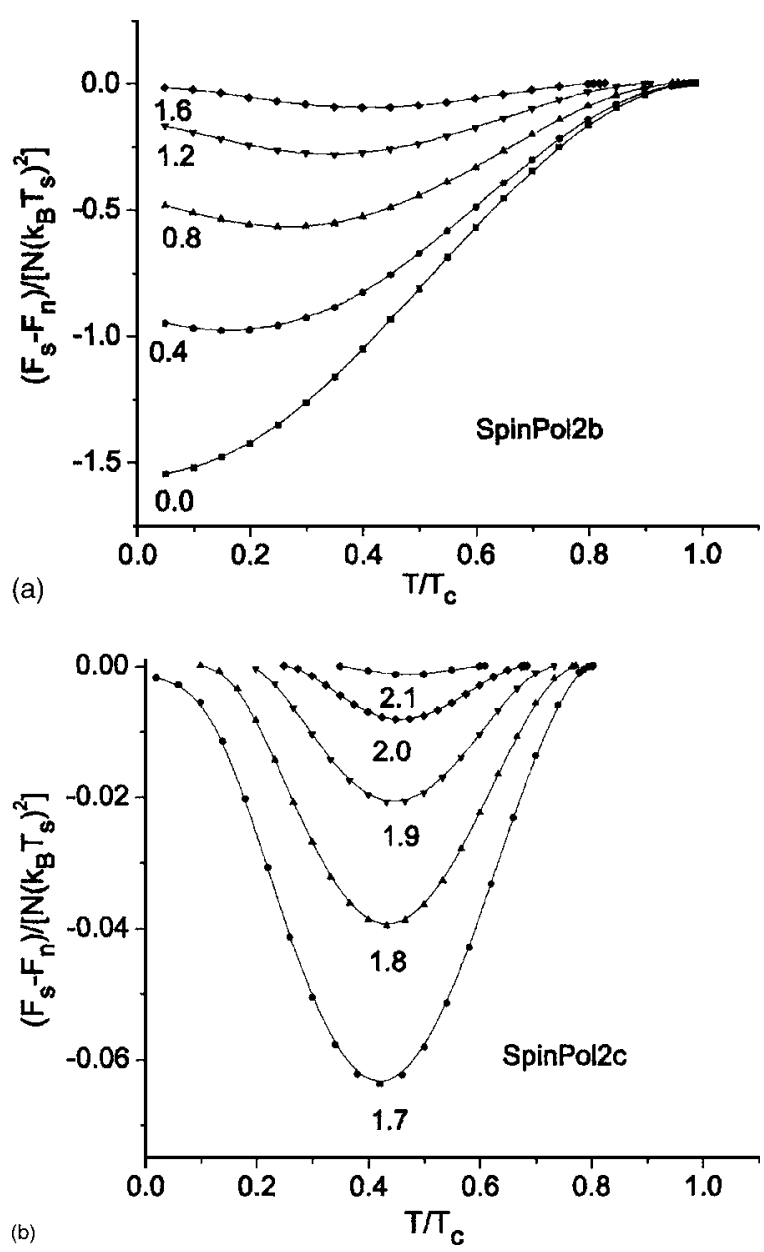

FIG. 3. (a) The free energy difference $\left(F_{s}-F_{n}\right) / N k_{B} T_{s}$ as a function of reduced temperature for different electron polarizations $\beta$ in the range of $0 \leqslant \beta \leqslant 1$.6. (b) The free energy difference $\left(F_{s}-F_{n}\right) / N k_{B} T_{s}$ as a function of reduced temperature for different electron polarizations $\beta$ in the range of $1.7 \leqslant \beta \leqslant 2.1$.

electrons differ by $2 \lambda$. This corresponds to a polarization of $m=2 \lambda N$ yielding $\lambda=\beta k_{B} T_{s} / 2$ and one obtains the condition

$$
\begin{aligned}
& \int_{0}^{k_{B} \Theta_{D}} \frac{1}{\varepsilon}\left[1-f\left(\frac{\varepsilon-\beta k_{B} T_{s} / 2}{k_{B} T_{m}}\right)-f\left(\frac{\varepsilon+\beta k_{B} T_{s} / 2}{k_{B} T_{m}}\right)\right] d \varepsilon \\
& \quad=\frac{1}{N V} .
\end{aligned}
$$

Combining this equation with the condition for $T_{s}$, one finds

$$
\int_{0}^{\infty} \frac{1}{x}\left[2 f(x t)-f\left(x+\frac{\beta}{2 t}\right)-f\left(x-\frac{\beta}{2 t}\right)\right] d x=0
$$

where $x=\varepsilon / k_{B} T_{m}, t=T_{m} / T_{s}$, and $\beta T_{s} / 2 T_{m}=\beta / 2 t$. Equation (5) is an implicit condition between $t$ and $\beta$ for the boundary between the normal and the superconducting state. In Fig. 4 the phase diagram is shown.

As a conclusion, we compare the two procedures to induce spin polarization in superconductors, high magnetic fields and spin injection. A type I superconductor tries to screen any external magnetic field until the field reaches the thermodynamic critical field $B_{c}$ where the superconductivity is destroyed. Since the interesting effects of spin polarization

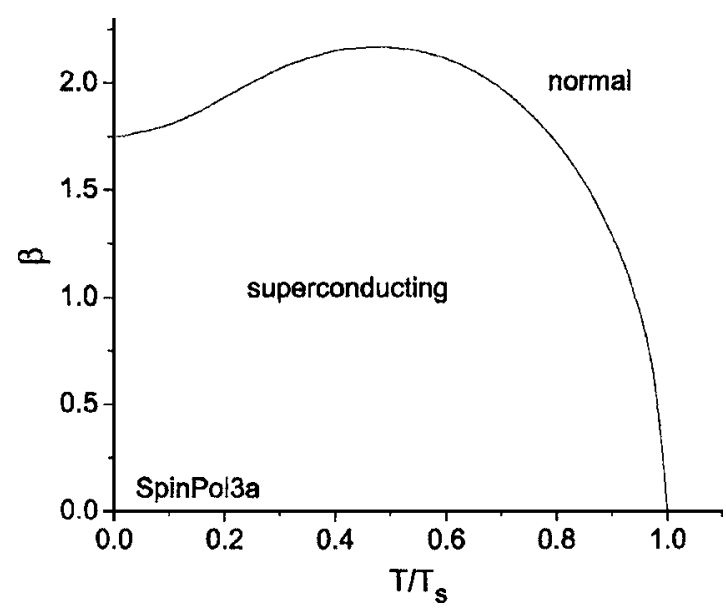

FIG. 4. The phase diagram in the temperature-polarization plane.

require a much larger magnetic field than $B_{c}$ one turns to thin films where the film thickness is much smaller than the magnetic penetration length $\lambda$. For example, Meservey and Tedrow used Al films with a thickness of $5 \mathrm{~nm}$. By using such thin films one has to pay the price that the mean free path of the conduction electrons is strongly reduced. This may have prevented, for example, observing the exotic Fulde-FerrelLarkin-Ovchinnikov (FFLO) superconducting state in highly polarized $s$ superconductors. In the ferromagnetsuperconductor-ferromagnet (FSF)-SETs one is not restricted by an external magnetic field. Once the magnetic electrodes are brought into the antiferromagnetic alignment the external magnetic field can be turned off. Therefore one can use thicker superconducting films with larger mean free path. This could improve the chances to observe the FFLO state in the highly polarized $\mathrm{Al}$ nanostructures. The combination of spin injection and nanosized islands promises an approach to investigate superconductors with high spin polarization.

${ }^{1}$ C. D. Chen, W. Kuo, D. S. Chung, J. H. Shyu, and C. S. Wu, Phys. Rev. Lett. 88, 047004 (2002).

${ }^{2}$ J. Johansson, M. Urech, D. Haviland, and V. Korenivski, Phys. Rev. Lett. 91, 149701 (2003).

${ }^{3}$ F. J. Jedema, H. B. Heersche, A. T. Filip, J. J. A. Baselmans, and B. J. van Wees, Nature (London) 416, 713 (2002).

${ }^{4}$ J. Johansson, M. Urech, D. Haviland, and V. Korenivski, J. Appl. Phys. 93, 8650 (2003).

${ }^{5}$ D. Wang and J. G. Lu, J. Appl. Phys. 97, 10A708 (2005).

${ }^{6}$ S. O. Valenzuela, D. J. Monsma, C. M. Marcus, V. Narayanamurti, and M. Tinkham, e-print cond-mat/0407489.

${ }^{7}$ S. Takahashi, H. Imamura, and S. Maekawa, Phys. Rev. Lett. 82, 3911 (1999).

${ }^{8}$ S. Takahashi, H. Imamura, and S. Maekawa, J. Appl. Phys. 87, 5227 (2000).

${ }^{9}$ A. M. Clogston, Phys. Rev. Lett. 9, 266 (1962).

${ }^{10}$ G. Sarma, J. Phys. Chem. Solids 24, 1029 (1963).

${ }^{11}$ K. Maki and T. Tsuneto, Prog. Theor. Phys. 31, 945 (1964).

${ }^{12}$ P. Fulde, Adv. Phys. 22, 667 (1973).

${ }^{13}$ R. Meservey and P. M. Tedrow, Phys. Rep. 238, 173 (1994).

${ }^{14}$ M. Tinkham, J. M. Hergenrother, and J. G. Lu, Phys. Rev. B 51, 12649 (1995).

${ }^{15}$ J. G. Lu, J. M. Hergenrother, and M. Tinkham, Phys. Rev. B 57, 120 (1998).

${ }^{16}$ J. G. Lu, J. M. Hergenrother, and M. Tinkham, Phys. Rev. B 53, 3543 (1996).

${ }^{17}$ N. Markovic, C. N. Lau, and M. Tinkham, Physica C 387, 44 (2003).

${ }^{18}$ J. Bardeen, L. N. Cooper, and J. R. Schrieffer, Phys. Rev. 108, 1175 (1957). 